\title{
Optimización y mejora del aprendizaje mediante la utilización de la realidad virtual en las prácticas de grados y ciclos formativos
}

\section{Ana Martínez Cuello, Bartolomé Serra Soriano, Encarna Piquer Maño, Pilar García Romero, Cristina Ribes Vallés y María Inmaculada Lloria Benet.}

aUCH-CEU ana.martinez1@uchceu.es, dUCH-CEU bartolome.serra@uchceu.es, bUCH-CEU encarna.piquer@uchceu.es, ${ }^{\circ} I S E P$ CEU CV pilar.garciaromero@.ciclosformativosceu.es, $\quad{ }^{\mathrm{E}} \mathrm{UCH}-\mathrm{CEU}$ cribes@uchceu.es, ${ }^{\text {f } U C H-C E U ~ m a r i a . l l o r i a @ u c h c e u . e s . ~}$

\begin{abstract}
Virtual reality has become a tool that opens up the possibilities of technology beyond its use for video games. Immersion in the real context in which events take place allows the ability of this technology to be extrapolated to the world of education to improve learning and adapt it to the demand of new generations. VR allows to locate the user within a virtual scenario that reproduces the corresponding learning environment. Through this project we seek to make available to students the practical content of the undergraduate subjects and vocational training modules, so that they can have it permanently without the need for face-to-face or a fixed schedule. The flexibility and ease of access to these contents also allows that for reasons of any kind (labor, personal, etc.) can track the practices without difficulties. The project includes both training cycle degrees (Dental Hygiene and Audiovisual Projects) and degree (Odontology and Architecture), showing the versatility and extension to the different sectors to which the use of this teaching methodology can reach.
\end{abstract}

Keywords: virtual reality, education, innovation, digitalization, learning.

\section{Resumen}

La realidad virtual se ha convertido en una herramienta que abre las posibilidades de la tecnología más allá de su uso para videojuegos. La inmersión en el contexto real en el que se desarrollan los acontecimientos permite extrapolar la capacidad de esa tecnología al mundo de la educación para mejorar el aprendizaje y adaptarlo a la demanda de las nuevas generaciones. La RV permite ubicar al propio usuario dentro de un escenario virtual que reproduce el entorno de aprendizaje correspondiente. Mediante este proyecto perseguimos poner a disposición de los alumnos el contenido práctico de las asignaturas de grado y los módulos de formación profesional, de manera que puedan disponer de él permanentemente sin necesidad de presencialidad ni de un horario fijo. La flexibilidad y facilidad de acceso a estos contenidos permite, además, que por motivos de cualquier indole (laborales, personales, etc.) puedan realizar el seguimiento de las prácticas sin ningún tipo de impedimento. El proyecto engloba tanto a titulaciones de ciclo formativo (Higiene Bucodental y Realización de Proyectos Audiovisuales y Espectáculos) como de grado (Odontología y Arquitectura), mostrando la versatilidad y la extensión a los diferentes sectores a los que puede llegar la utilización de esta metodología docente.

Palabras clave: realidad virtual, educación, innovación, digitalización, aprendizaje. 


\section{Introducción}

Jamás se produjeron tantas innovaciones disruptivas en tan corto período de tiempo como ahora en la sociedad digital. Nos movemos en un mundo líquido, flexible y voluble, que Bauman contrapone al tiempo sólido, estable, repetitivo y lleno de certezas al que estábamos acostumbrados.

Por su parte, se hace necesario invertir en capital humano con el fin de que los trabajadores adquieran las nuevas competencias que va a demandar el mercado laboral. Las disrupciones, las ya producidas y las que vendrán, nos afectan a todos, a nuestro estilo de vida y también a nuestra forma de trabajar. Para estas situaciones y circunstancias complejas, ambiguas, inciertas y vulnerables, se hace preciso cambiar las formas de educar, integrando las nuevas herramientas tecnológicas en los procesos educativos, con el fin de acometer profundos cambios tanto metodológicos como organizativos en nuestro sistema educativo. Pero es que, además, nuestros niños, adolescentes y jóvenes son, también, radicalmente diferentes a los adultos. Ahí nos encontramos con conceptos como los de nativo y residente digitales, generaciones X, Y y $\mathrm{Z}$ referidos a esos estudiantes que pueblan el sistema. Así, resultaría incomprensible que, en una sociedad digital con estudiantes de esas generaciones, no arbitrásemos modelos educativos digitales, aprendizajes digitales. Si la educación actual no se integra en la realidad digital que nos que nos circunda, otros actores ocuparán su lugar. (García, 2019).

Gracias al desarrollo de las nuevas tecnologías, el potencial de la formación a distancia ha sufrido un avance muy importante en los últimos años incrementando las facilitando las posibilidades de educación a distancia. En la actualidad hay numerosas metodologías educativas en sistemas convencionales, fichas prácticas, diapositivas, vídeos...

Basándonos en el concepto de realidad virtual (RV), donde un entorno de escenas u objetos de apariencia real crea al usuario la sensación de estar inmerso en él, consideramos este sistema como una excelente metodología educativa adicional para aquellos estudiantes que motivos diversos no pueden acudir a las aulas.

En la evolución de la humanidad uno de los sentidos que más ha repercutido es el visual. La realidad en la que nos encontramos está envuelta en continuos reclamos visuales. El soporte mediante el cual se transmite está en las TIC (Tecnologías de la Información y de la Comunicación). Es un mundo en constante evolución y adaptación siendo una muestra de ello la realidad virtual. La adecuación tecnológica ha hecho que se extrapole al ámbito educativo, fomentando la autonomía y la creatividad del alumno. También la necesidad de adecuarse a nuevos escenarios y papel más activo en binomio enseñanza- aprendizaje (Lafarga, 2017).

La base de la metodología utilizada es la realidad virtual inmersiva para que el alumno pueda experimentar la sensación de estar dentro del aula durante la realización de la práctica (Otero,2011). Para ello solo es necesario disponer de unas gafas de RV (de bajo coste) y un móvil con conexión a internet. El sistema operativo (Android o IOS) es independiente, pues la plataforma de visualización se puede desarrollar para ambos sistemas.

Además, la realidad virtual además en las disciplinas relacionadas con la salud proporciona un entorno real que puede hacer sentir las emociones y sensaciones físicas como si estuvieran allí, la sensación de tener un paciente real, el instrumental necesario, y la disposición espacial del lugar de trabajo (Ortega, 2018).

Según el equipo de All VR Education, que colabora en esta materia con el Ministerio de Educación, Cultura y Deporte de España, algunas de las posibilidades de la realidad virtual son:

- Mostrar a los alumnos, en primera persona un procedimiento "in situ", por ejemplo, un experimento o proyecto.

- Experimentar en primera persona la representación de un acontecimiento

(c)) EY-NC-ND 2020, Universitat Politècnica de València

Congreso In-Red (2020) 
- Visitar virtualmente cualquier parte del mundo real o ficticia.

- Vivir experiencias inmersivas.

Con esto no se pretende menospreciar el uso de instrumentos de lectura, si no reforzar los contenidos aprendidos (Bautista, 2017).

Operativamente, una vez se ha planificado, grabado y editado la práctica, el proceso es muy sencillo: el móvil se introduce en las gafas y al ponérselas el alumno "entra" en el espacio donde se desarrolla la práctica, pudiendo observar el ejercicio como si estuviera al lado del profesor. Dentro de la práctica, el alumno podrá ver planos de detalle del campo de trabajo (zoom), el guion virtual escrito con los materiales que se utilicen, el proceso/explicación de lo que se esté ejecutando, así como los resultados de la propia práctica. Esto hace que, al mismo tiempo que se mejora la competencia de aprendizaje práctico del alumnado, se fomenten las competencias digitales imprescindibles en la sociedad de la información actual (Gisbert, 2016).

\section{Objetivos}

El objetivo primordial de este proyecto pionero se basa en intentar sistematizar la aplicación de la RV en varias disciplinas, ajustarla a la dinámica de diferentes modalidades de enseñanza y a un calendario para, posteriormente, extraer conclusiones para fases posteriores e, incluso, otras disciplinas o niveles formativos. Además, como objetivo secundario se persigue el avanzar en la formación personalizada y flexible del alumnado, de manera que pueda sumergirse de manera virtual en un entorno real del campus (un gabinete dental, una clínica odontológica, un aula de prácticas, un aula de exposición, un plató de grabación, etc.) como si estuviera presenciando la práctica en vivo, pero de una forma más enriquecida al incorporar realidad extendida. Con ello el proceso formativo-educativo se mejora, y cualquier alumno, nacional o internacional, desde cualquier lugar del mundo puede estudiar y repetir tantas veces como desee las correspondientes prácticas (González 2011), lo que permite compaginar el trabajo u otras ocupaciones con los estudios y haciendo más fácil y asequible el aprendizaje. Además, de ese modo también se optimiza el gasto repetido del posible material utilizado para la ejecución de las prácticas y se satisface la necesidad de atención 24/7/365 que tanto demandan los alumnos de la actual "sociedad de la inmediatez".

Inmersos en los objetivos de este trabajo se nos presentan varias preguntas interesantes:

- ¿Por qué no crear escenarios sanitarios simulados para practicar de forma dinámica, interactiva, científica y segura sin salir de la comodidad de nuestra vivienda?

- ¿Por qué no crear un recurso educativo con la realidad virtual para ayudar a los estudiantes de las Ciencias de la Salud y también de otro tipo de disciplinas?

- ¿Cómo generar procesos de aprendizaje significativo dentro y fuera del aula de clases?

Además, a la luz de los últimos acontecimientos sociales a nivel mundial con la pandemia del COVID-19 que imposibilita de modo temporal la presencialidad en las clases teóricas y prácticas y obliga a la digitalización de las clases, el alumnado puede continuar con su formación práctica, comprendiendo así procedimientos que sólo de un modo teórico son difíciles de visualizar e interiorizar; aumentando así la calidad docente en modalidades no presenciales. 


\section{Desarrollo de la innovación}

El desarrollo de este proyecto de innovación docente se inició en febrero de 2018 con una fase de planificación en la que se designaron las prácticas que iban a ser objeto de grabación con cámara $360^{\circ}$. Las mismas, surgen de aquellos módulos que cuentan con una parte práctica dentro del primer curso del Ciclo Superior de Higiene Bucodental. Los módulos anteriormente mencionados son Intervención bucodental, Exploración de la cavidad oral y Fisiopatología general.

A continuación, se definieron los materiales con los que se debía contar para cada uno de los procedimientos prácticos y el espacio (gabinete odontológico, aula de simulación preclínica, laboratorio o sala de disección) en el que se desarrollaría los mismos. Por último, en esta fase, se seleccionó a los docentes implicados en las grabaciones y se les designó cada uno de los procedimientos prácticos a desarrollar.

En el periodo restante del mes de febrero el equipo docente implicado desarrolló un guion escrito en el que se le otorgaba una numeración y un título a cada una de las prácticas, el espacio donde se debía desarrollar, un breve resumen del procedimiento y los materiales requeridos para poder coordinar al equipo docente, el personal implicado en abastecer los materiales, la reserva de espacios y el equipo de grabación.

En los meses comprendidos entre marzo y abril de 2019 se llevó a cabo la grabación de los distintos procedimientos de los módulos implicados. Para tal efecto, se designó un calendario de grabación para cada docente, se reservó el espacio adecuado para el desarrollo del procedimiento y se elaboró una preparación previa del material a utilizar. En algunas ocasiones, contamos la colaboración de alumnos de la universidad para ejercer como personal auxiliar durante el procedimiento o como paciente. Para ello, los alumnos participantes firmaron un documento de cesión de derecho de imagen.

Durante la grabación, además de la cámara $360^{\circ}$, se realizó un plano detalle de algunos procedimientos que necesitan ser vistos con mayor aumento para el alumno. De este modo, en el proceso de edición de cada procedimiento, se incluyó una proyección superpuesta con los planos de detalle del campo de trabajo (zoom), el guion virtual escrito con los materiales que se utilizan, el proceso/explicación de lo que se está ejecutando, así como los resultados de la propia práctica.

Una vez grabadas y editadas las prácticas, se procedió a la comprobación de cada uno de los vídeos por parte del equipo docente para revisar la concordancia en cuanto a título, procedimiento, materiales especificados, planos detalles, narración del proceso, cronología, etc. durante los meses de junio y julio de 2019.

Tabla 1. Planificación temporal curso 2018-2019

\begin{tabular}{|c|c|c|c|c|c|c|c|c|c|c|}
\hline FASE/ACTIVIDAD/TAREA & \multicolumn{10}{|c|}{ Duración Temporal Curso 18-19 } \\
\hline \multirow{2}{*}{$\begin{array}{l}\text { Planificación de las prácticas (materiales, espacios y } \\
\text { personas implicadas) }\end{array}$} & Dic & En & Feb & Mar & $A b$ & May & $\mathrm{Ju}$ & Jul & $\mathrm{Ag}$ & Sep \\
\hline & & & $\mathbf{x}$ & & & & & & & \\
\hline \multirow{2}{*}{ Elaboración del guion escrito de las prácticas } & Dic & En & Feb & Mar & $A b$ & May & Ju & Jul & $\mathrm{Ag}$ & Sep \\
\hline & & & $\mathbf{x}$ & & & & & & & \\
\hline \multirow{2}{*}{ Grabación y edición de las prácticas } & Dic & En & Feb & Mar & $A b$ & May & Ju & Jul & $\mathrm{Ag}$ & Sep \\
\hline & & & & $\mathbf{x}$ & $\mathbf{x}$ & & & & & \\
\hline \multirow{2}{*}{ Comprobación, revisión y ajustes } & Dic & En & Feb & Mar & $A b$ & May & Ju & Jul & $\mathrm{Ag}$ & Sep \\
\hline & & & & & & & $\mathbf{x}$ & $\mathbf{x}$ & & \\
\hline
\end{tabular}


En septiembre de 2019, coincidiendo con el inicio del nuevo curso presente, se comenzó con la implementación del visionado de los vídeos de RV tanto en la modalidad presencial como en la modalidad semipresencial. Para ello se citó a todos los alumnos de ambas modalidades en el aula para repartirles las gafas de visualización de vídeos en formato 3D y explicarles el funcionamiento de estas y de la plataforma donde pueden descargar cada uno de los procedimientos prácticos. Para ello, nuestro equipo realizó un vídeo explicativo en el que se les indicaba los pasos a seguir para acceder a la plataforma y seleccionar el vídeo en cuestión que se proyectó en el aula. Una vez interiorizado el proceso de visualización, instamos a los alumnos al visionado de cada una de las prácticas (según el cronograma del curso facilitado al inicio de este en el que se detallan cada una de las sesiones teóricas y prácticas y la fecha del desarrollo de estas) previo a la realización de estas. Del mismo modo, se les pidió a los alumnos que participasen activamente en el proceso de comprobación y revisión de los vídeos y visionado de los mismos trasladándonos cualquier incidencia o dificultad que percibiesen.

En los meses de enero y febrero, una vez transcurridas las primeras visualizaciones previas a la realización de las prácticas in situ para los alumnos de la modalidad presencial y como herramienta de refuerzo docente ante el desarrollo teórico de los distintos procedimientos para la modalidad semipresencial, se ha procedido a la realización de cuatro cuestionarios mediante la herramienta Microsoft Forms en los que sea abordan 4 temáticas: la usabilidad de la plataforma, los resultados académicos, el nivel de mejora docente y el nivel de aprendizaje previo a la realización de la práctica "in situ".

El primero de los cuestionarios, que alude a la usabilidad de la plataforma, consiste en 6 cuestiones (algunas cerradas y otras abiertas) que hacen referencia al acceso a la plataforma en el último mes, la utilidad para el repaso y seguimiento de las prácticas, experiencias previas con la Realidad Virtual y el área, aspectos a destacar en esta metodología docente y valoración de esta.

En el segundo cuestionario acerca de los resultados académicos se plantean 5 cuestiones aludiendo a la valoración de sus resultados académicos, la efectividad y productividad de la realización de las prácticas tras su visualización, la relación entre los resultados y la visualización, el nivel alcanzado y el valor añadido aportado por ISEP CEU.

En el tercer cuestionario, el que trata de evaluar el nivel de mejora de docencia, está formado por 5 preguntas en las que se cuestiona si los vídeos ayudan a mejorar las prácticas clínicas o a preparar mejor los exámenes, si sirven para repasar las prácticas realizadas volviendo a verlos tras las mismas, si les resultaría práctico disponer de los vídeos del primer curso en el segundo también y si consideran que esta metodología docente es innovadora y diferencial.

Por último, el cuarto de los cuestionarios está planteado para obtener el feed-back acerca del nivel de aprendizaje adquirido previo a la realización de las prácticas de modo presencial. Para ello se proponen 6 cuestiones en las que se pregunta si la visualización previa ha ayudado a plantear menos dudas, a tener una mejor preparación y más claros los conceptos; también se pregunta acerca del nivel de aprendizaje previo adquirido y a si incrementa la capacidad de recordar procedimientos, la seguridad ante la práctica real y la velocidad en la consecución de estas. 
Tabla 2. Planificación temporal curso 2019-2020

\begin{tabular}{|c|c|c|c|c|c|c|c|c|c|c|}
\hline FASE/ACTIVIDAD/TAREA & \multicolumn{10}{|c|}{ Duración Temporal Curso 19-20 } \\
\hline \multirow{2}{*}{ Implementación en las clases } & Sep & Oct & Nov & Dic & En & Feb & Mar & $\mathrm{Abr}$ & May & Jun \\
\hline & $\mathbf{x}$ & $\mathbf{x}$ & $\mathbf{x}$ & $\mathbf{x}$ & & & & & & \\
\hline \multirow{2}{*}{$\begin{array}{l}\text { Realización de encuestas ad-hoc sobre el proyecto de } \\
\text { RV }\end{array}$} & $\overline{\text { Sep }}$ & $\overline{\text { Oct }}$ & Nov & Dic & $\overline{\text { En }}$ & $\overline{\text { Feb }}$ & Mar & $\overline{A b r}$ & May & Jun \\
\hline & & & & & $\mathrm{x}$ & $\mathbf{x}$ & & & & \\
\hline \multirow{2}{*}{ Análisis de resultados y reporte } & Sep & Oct & Nov & Dic & En & Feb & Mar & $\mathrm{Abr}$ & May & Jun \\
\hline & & & & & & & $\mathbf{x}$ & $\mathbf{x}$ & $\mathrm{x}$ & \\
\hline \multirow{2}{*}{ Desarrollo de mejora y actualización de contenidos } & Sep & Oct & Nov & Dic & En & Feb & Mar & Abr & May & Jun \\
\hline & & & & & & & & & & $\mathrm{x}$ \\
\hline
\end{tabular}

Simultáneamente a este proceso, en el curso presente hemos empezado con el proceso de planificación y grabación de los vídeos correspondientes a las prácticas y procedimientos clínicos, preclínicos y de laboratorio del segundo curso. Los módulos que cuentan con prácticas son Conservadora, Periodoncia, Cirugía e Implantes; Prótesis y Ortodoncia y Primeros Auxilios.

En octubre de 2019 planificamos las prácticas que iban a desarrollarse, los espacios que necesitábamos reservar y el equipo docente implicado. En los sucesivos meses, noviembre y diciembre de 2019, elaboramos el guion escrito para cada una de las prácticas detallando cada procedimiento.

Desde enero de este año estamos grabando los vídeos pertenecientes al segundo curso del ciclo. Nuestra previsión era terminar la grabación entre marzo y abril y durante los meses de verano insertarlo y codificarlo en la plataforma Blackboard. Pero debido a la circunstancia actual que vivimos con la pandemia del COVID-19 y dado el confinamiento indefinido al que estamos sometidos, con toda seguridad este proceso se vea alterado.

Tabla 3. Planificación temporal curso 2019-2020

\begin{tabular}{|l|l|l|l|l|l|l|l|l|l|l|l|}
\hline \multicolumn{2}{|c|}{ FASE/ACTIVIDAD/TAREA } & \multicolumn{5}{|c|}{ Duración Temporal Curso 19-20 } \\
\hline $\begin{array}{l}\text { Planificación de las prácticas (materiales, espacios y } \\
\text { personas implicadas) }\end{array}$ & Oct & Nov & Dic & En & Feb & Mar & Ab & May & Ju & Jul \\
\cline { 2 - 10 } & $\mathbf{x}$ & & & & & & & & \\
\hline Elaboración del guion escrito de las prácticas & Oct & Nov & Dic & En & Feb & Mar & Ab & May & Ju & Jul \\
\hline & & $\mathbf{x}$ & $\mathbf{x}$ & & & & & & & \\
\hline Grabación y edición de las prácticas & & Oct & Nov & Dic & En & Feb & Mar & Ab & May & Ju & Jul \\
\hline & & & & $\mathbf{x}$ & $\mathbf{x}$ & $\mathbf{x}$ & $\mathbf{x}$ & & & \\
\hline Inserción en Blackboard y codificación & & Oct & Nov & Dic & En & Feb & Mar & Ab & May & Ju & Jul \\
\hline
\end{tabular}

Nuestro objetivo era empezar a implementarlo en las clases a partir de septiembre, en enero realizar las encuestas ad-hoc sobre los vídeos de RV, analizar los resultados y dar reporte de estos en febrero para 
establecer un programa de mejora y de actualización de contenidos en los meses comprendidos entre marzo y mayo de 2021.

Tabla 4.. Planificación temporal curso 2020-2021

\begin{tabular}{|c|c|c|c|c|c|c|c|c|c|}
\hline FASE/ACTIVIDAD/TAREA & \multicolumn{9}{|c|}{ Duración Temporal Curso 20-21 } \\
\hline \multirow{2}{*}{ Implementación en las clases } & Sep & Oct & Nov & Dic & En & Feb & Mar & Abr & May \\
\hline & $\mathbf{x}$ & $\mathbf{x}$ & $x$ & $\mathbf{x}$ & & & & & \\
\hline \multirow{2}{*}{$\begin{array}{l}\text { Realización de encuestas ad-hoc sobre el proyecto de } \\
\text { RV }\end{array}$} & Sep & Oct & Nov & Dic & En & Feb & Mar & Abr & May \\
\hline & & & & & $\mathbf{x}$ & & & & \\
\hline \multirow{2}{*}{ Análisis de resultados y reporte } & Sep & Oct & Nov & Dic & En & Feb & Mar & $\mathrm{Abr}$ & May \\
\hline & & & & & & $\mathbf{x}$ & & & \\
\hline \multirow{2}{*}{ Desarrollo de mejora y actualización de contenidos } & Sep & 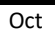 & Nov & 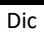 & En & Feb & Mar & $\mathrm{Abr}$ & May \\
\hline & & & & & & & $\mathbf{x}$ & $\mathbf{x}$ & $x$ \\
\hline
\end{tabular}

\section{Resultados}

Siguiendo con la temporalización fijada en el desarrollo del proyecto de innovación docente nos encontramos en el período de realización de encuestas ad-hoc sobre el visionado de los vídeos de Realidad Virtual al alumnado implicado.

Hasta la fecha hemos pasado el primer cuestionario referente a la usabilidad de la plataforma. El mismo ha sido contestado por 18 de los 19 alumnos que componen el primer curso del Ciclo Superior de Higiene Bucodental de ISEP CEU en Valencia: 17 alumnos de modalidad presencial y 2 alumnos de modalidad semipresencial. El tiempo medio dedicado a realizar el mismo ha sido de 2 minutos y 40 segundos.

A la primera cuestión de la encuesta “¿Cuántas veces has accedido a los vídeos VR 360 en el último mes?”, 5 alumnos contestaron que ninguna vez, 9 alumnos marcaron la opción entre 1 y 5 veces, 4 alumnos lo hicieron entre 6 y 10 veces y ningún alumno lo hizo más de 10 veces (Figura 1).

\section{Visualizaciones el último mes}

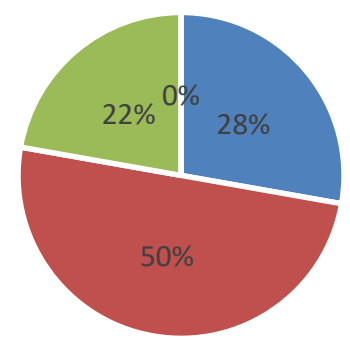

- Ninguna - Entre 1 y 5 Entre 6 y 10 - Mas de 10

Fig. 1. Resultados $1^{a}$ cuestión $1^{o}$ Feb_Cuestionario VR 360 Higiene Bucodental_2019 
A la segunda pregunta “¿Cómo te han resultado de útiles para el seguimiento y repaso de las prácticas? ”, 3 alumnos contestaron que poco, 11 alumnos se declinaron por la opción algo y a 4 les parecía de mucha utilidad (Figura 2).

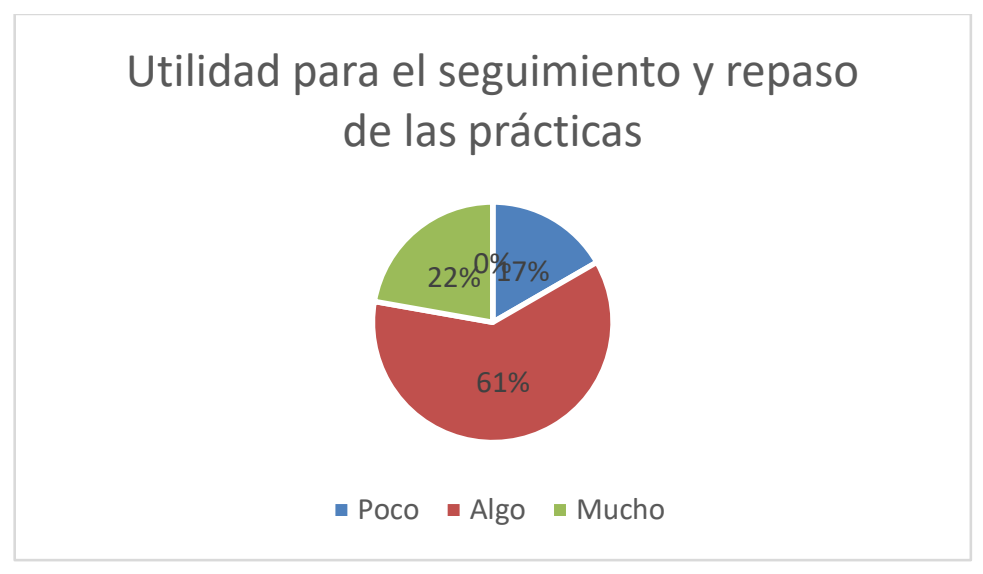

Fig. 2. Resultados $2^{a}$ cuestión $1^{o}$ Feb_Cuestionario VR 360 Higiene Bucodental_2019

A la tercera pregunta “¿Qué destacarías de esta nueva metodología docente?” encontramos variadas respuestas del alumnado y en general la consideran interesante, dinámica, entretenida, una nueva forma de aprender y que brinda la oportunidad de repasar conceptos en casa, innovadora, permite visualizar detalles, complementa las prácticas y permite la repetición del visionado de estas.

A la cuarta cuestión “¿Habías utilizado antes un dispositivo de Realidad Virtual?” 17 alumnos no lo habían hecho anteriormente y 1 alumno sí (Figura 3).

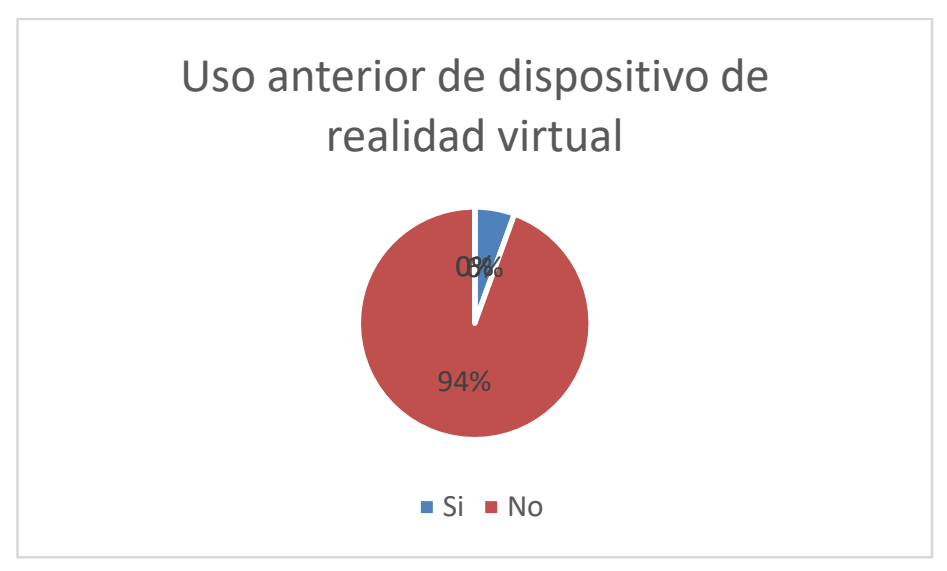

Fig. 3. Resultados $4^{a}$ cuestión $1^{o}$ Feb_Cuestionario VR 360 Higiene Bucodental_2019

En la quinta pregunta, la cual debían contestar los alumnos que eligiesen en la anterior pregunta la respuesta afirmativa, “¿Podrías comentar tu experiencia”? el alumno con experiencia previa comentó que tuvo una experiencia mejorable. 
En la sexta pregunta “CCómo valoras la utilización de la Realidad Virtual en tu formación?”, 1 alumno la consideró imprescindible, 8 alumnos la consideraron muy positiva y adaptada a la actualidad, otros 8 alumnos la consideran una herramienta más y 1 la considera novedosa pero no aporta nada (Figura 4).

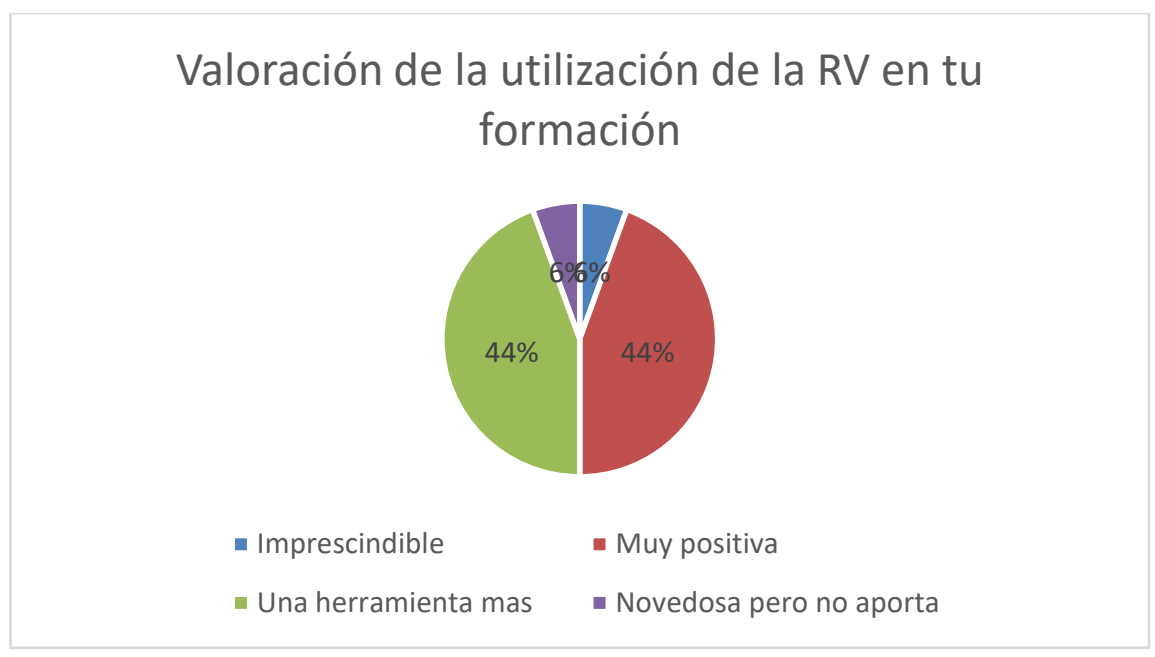

Fig. 4. Resultados $6^{a}$ cuestión $1^{\circ}$ Feb_Cuestionario VR 360 Higiene Bucodental_2019

Por último, en la séptima cuestión “¿Crees que la Realidad Virtual mejora y flexibiliza tu manera de aprender?" 15 alumnos contestaron de un modo afirmativo frente a 3 alumnos que lo hicieron de negativamente (Figura 5).

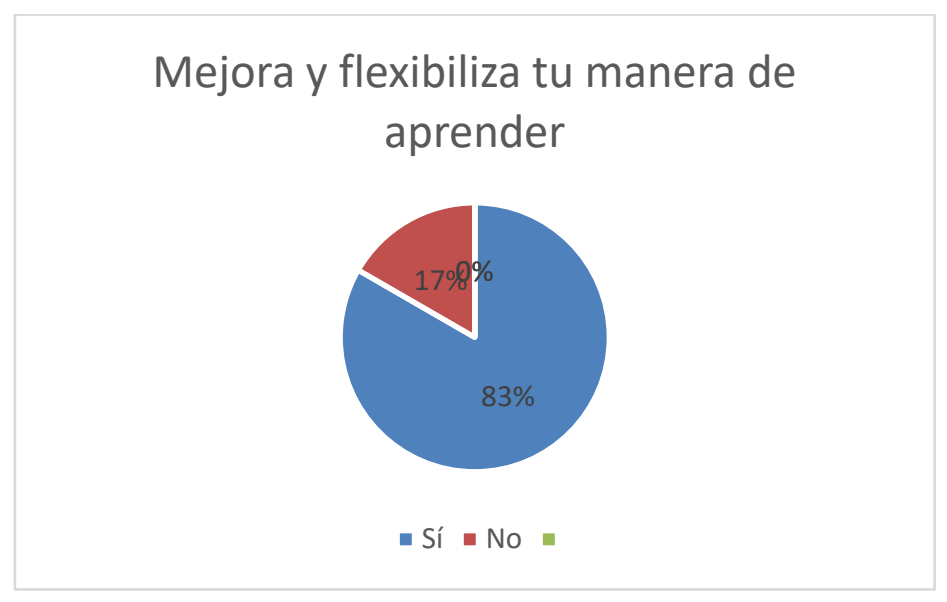

Fig. 5. Resultados $7^{a}$ cuestión $1^{o}$ Feb_Cuestionario VR 360 Higiene Bucodental_2019 


\section{Conclusiones}

Es importante mencionar los distintos conocimientos concluidos, tras la realización de este estudio de RV con alumnos de ciclos formativos:

En relación con nuestra primera pregunta mostrada en los objetivos iniciales, la conclusión en cuanto a la formación de escenarios sanitarios simulados para prácticas es que es positiva y enriquecedora. Es muy importante el acondicionamiento inicial del contexto en el que se han de integrar las sesiones de RV. Éste es un factor clave para el éxito, pero no es el único. Por eso hay que mencionar la necesidad del trabajo en el cambio o modelación de las competencias de los docentes (formación específica, actualización constante de conocimientos, adaptación de horarios, etc.). También hay que tener en cuenta exigencias de planificación y preparación de las sesiones de grabación, teniendo en cuenta el nivel de experiencia de cada docente.

Por ello, hemos llegado a la conclusión de que es muy conveniente realizar un estudio de necesidades y dificultades antes, durante y al final de cada proyecto.

En cuanto a nuestro objetivo de conseguir aprendizajes significativos con nuestro proyecto de RV, hemos comprobado que el estudiante es una pieza clave dentro de este engranaje. El alumno debe identificar su estilo de aprendizaje y cuáles son sus características para procesar la información. El sistema de RV permite al estudiante recibir material y seguir de manera personalizada los vídeos que satisfagan sus preferencias. Así se potencia que el alumno pueda aprender de forma autónoma, al orientar su propio conocimiento y que esto favorezca al desarrollo de competencias de gestión de aprendizaje. Sería interesante hacer una biblioteca con recursos de Realidad Virtual con material de consulta recurrente, para que el alumno afiance conceptos.

Este sistema de RV potencia la interacción profesor-estudiante, ya que provee de posibilidades de retroalimentación inmediata acerca de las fortalezas y debilidades del estudiante, centrándose así en las necesidades individuales de cada uno.

No obstante, necesitamos continuar con la recolección de resultados para establecer unas conclusiones definitivas ante la implementación de esta metodología de innovación docente, ya que nos hallamos en la fase inicial de esta etapa de recolección de estos. 


\section{Referencias}

- BAUtista, P., HeRranz de la CASA, J. M., Y CANTERO DE JULiÁN, J. I. (2017) "Comunicación y educación en formato 360 y realidad virtual" en Senderos de historia cultural. ISBN 978-980-247-265-9, págs. 149-178.

- García Aretio, L. (2019) "Necesidad De Una Educación Digital En Un Mundo Digital." Revista Iberoamericana De Educación a Distancia 22.2 (2019): 9-22.

- Gisbert Cervera, M., GonzÁlez Martínez, J., Y Esteve Mon, F. M. (2016) "Competencia digital y competencia digital docente: una panorámica sobre el estado de la cuestión" en Revista Interuniversitaria de Investigación En Tecnología Educativa, 0(0). https://doi.org/10.6018/riite2016/257631

- GonzÁlez AsperA, A., \& ChÁvez HernándEZ, G. (2011). "La realidad virtual inmersiva en ambientes inteligentes de aprendizaje. Un caso en la educación superior". Revista ICONO14 Revista Científica De Comunicación Y Tecnologías Emergentes, 9(2), 122-137. https://doi.org/10.7195/ri14.v9i2.42

- Lafarga Ostáriz, P., Fuentes CABrera, A. Y Romero RodríGUeZ, J. M. (2017) La realidad virtual, el futuro visual de la educación. Eindhoven: Redine.

- Ortega Caballero, i. Ortega Caballero, A. (2018). "La realidad virtual como agente" en Transmisión del Conocimiento Educativo y de la Salud. (2018). ISSN-e 19896247, Extra 1, 2018, págs. 439-458.

- Otero Franco, A., \& Flores GonzÁlez, J. (2011). "Realidad virtual: Un medio de comunicación de contenidos" en Aplicación como herramienta educativa y factores de diseño $e$ implantación en museos y espacios públicos. Revista ICONO14 Revista Científica De Comunicación Y Tecnologías Emergentes, 9 (2), 185-211. https://doi.org/10.7195/ri14.v9i2.28 